\title{
Virtual Care: The Future for Telehealth
}

\author{
Lua PERIMAL-LEWIS ${ }^{\mathrm{a}, 1}$ Patricia A.H. WILLIAMS ${ }^{\mathrm{a}, \mathrm{b}}$ Ginger MUDD $^{\mathrm{a}, \mathrm{b}}$ \\ and Gihan GUNASEKARA ${ }^{\mathrm{a}, \mathrm{b}}$ \\ ${ }^{a}$ Flinders Digital Health Research Centre, Flinders University, Adelaide, Australia \\ ${ }^{\mathrm{b}}$ Cisco-Flinders Digital Health Design Lab, Flinders University, Adelaide, Australia
}

\begin{abstract}
The COVID-19 pandemic has brought telehealth into the limelight. Telehealth is not a new word but since the pandemic, for many day-to-day users of the healthcare system, this term has become a household term. For IT enthusiasts, it is refreshing to see the uptake in telehealth. In most cases the uptake in telehealth came from the forced necessity of minimizing the spread of the virus. The positive outcomes have taken healthcare by surprise. It is not surprising then to see healthcare service providers transitioning to telehealth at an exponential rate. However, the upcoming COVID-19 normal state will demand more than the transitioning of 'brick and mortar' clinical practices to video or tele consult, making telehealth a natural predecessor of virtual care. This is a position paper presenting the current state of telehealth by outlining its benefits, limitations, looking beyond telehealth to address some of the recurring healthcare pain points and potential solutions to move towards patient-centered care via the adoption of virtual care.
\end{abstract}

Keywords. Telehealth, virtual care, healthcare pain points

\section{Introduction}

Australia's health system is one of the best in the world, providing safe and affordable health care for all Australians. COVID-19 has forced the health system to rapidly embrace telehealth practices and technologies. In most cases telehealth is simply transferring what used to happen in person (e.g. a consultation with a health professional) to an online format to create a short-term workaround. However, stop gap solutions mean patient experience can be poor, unengaging, sessions may be insecure, and data potentially compromised. In the current pandemic, the technology rather than the medical interaction and advice has become the focus. Therefore, there is an urgent need for the health sector to move away from telehealth to the broader concept of virtual care, where a broader range of health activities can be virtual.

Australian healthcare needs innovation and virtual care is one powerful modernization that does not require dramatic disruption to our existing systems. Virtual care does enhance, improve and complement traditional care. The term "virtual care" is an umbrella term [1] for a wide variety of terms that are used to describe the various ways and means that health care may be provided from a distance. Virtual care encompasses the commonly used terms of telehealth and telemedicine (defined below) yet is broader and not limited by these terms:

\footnotetext{
${ }^{1}$ Lua Perimal-Lewis, Corresponding author, Flinders Digital Health Research Centre, College of Science \& Engineering, Flinders University, GPO Box 2100 Adelaide SA 5001, Australia; E-mail: lua.perimallewis@flinders.edu.au.
} 
i. telehealth - the use of telecommunication techniques for the purpose of providing telemedicine, medical education and health education over distance $[2,3]$

ii. telemedicine - use of advanced telecommunication technologies to exchange health information and provide health care services across geographic, time, social and cultural barriers $[2,4]$

The purpose of virtual care is to improve our healthcare systems - that is the "organization of people, institutions, and resources that deliver health care services to meet the health needs of people" [5] whilst constraining the ever-increasing costs of healthcare. Virtual care is the delivery of health care over a distance using various forms of communication or information technologies $[6,7]$ and where traditional face-to-face care delivery is complemented with technology enabled remote care. Virtual care may be from clinician to patient, patient to mobile technology or healthcare provider to healthcare provider. Hence, virtual care is a broad term for all the digital tools and realtime communication, to enable health providers to remotely interact with the patient.

Australia is well placed to advance virtual care, with the maturing adoption of electronic health records including the national MyHealth Record facilitating access to patient health information, with permission, from anywhere. Interoperability of electronic health records is an essential component of virtual care and enables integration of communication platforms and patient health information within virtual consultations.

This position paper views virtual care through a wide lens by considering continuity of care outside of acute care environments, e.g. at home, in aged care facilities, and primary care. It provides insight into common healthcare pain points that could be addressed by looking beyond telehealth.

\section{Benefits of telehealth}

There are numerous benefits of moving to the virtualization of communication that telehealth provides. The most obvious and well reported is that expansion of access to care and the move to a more on-demand model convenient to the patient [8]. Indeed, disease specific examples of the success of telehealth, for instance in cancer care [9] demonstrate the benefit in patient monitoring and management for earlier detection of complications. This in turn leads to better patient outcomes and lower costs from reduced hospitalizations [10].

For health professionals, telehealth visits are more efficient [11] and help doctors make use of unused time and see additional patients [12] which in turn brings a higher income for private practices. The convenience of being able to attend a consultation without the need to travel to a health facility means the likelihood of no-shows or cancellation of appointments by a patient is lower, enabling better efficiency for the practice.

Improved efficiency will have flow on effect for patients, health professionals and the overall healthcare system. This will contribute improved patient satisfaction, improved relationship between all parties involved in the care pathway, improved quality of care as a consequence of operating in a less chaotic environment, improves overall productive within the healthcare sector, enable innovation and more importantly; it will enable availability, accessibility and equitable healthcare. 


\section{Limitation of telehealth}

What is urgently needed is to redefine telehealth so that it is broader, more data-intensive and ultimately designed to deliver a superior patient and clinician experience and outcomes. Whilst telehealth has been with us in Australia for some time, particularly for remote healthcare, it is vastly underutilized and not broadly incentivized by our healthcare funding models. The predominant issue is the lack of widespread use and adoption of telehealth.

The need to improve poor health outcomes in rural and remote communities in Australia is well recognized. Whilst telehealth and telemedicine have been widely used in Australia over the past decade as a means of overcoming problems of access to health care and the shortage of health professionals in rural and remote areas [13], in many cases, telemedicine and telehealth are used to augment other service delivery models [14]. Effective and patient-centred care requires patient engagement, resource and care coordination, and a "new" patient-physician relationship [15]. Where telehealth replaces the consultation element, it does not encompass a holistic experience for the patient. The barriers that telehealth addresses, particularly for rural and remote areas, are a huge step in reducing the inequity in access to healthcare services [16]. However, many of the barriers that exist with lack health providers, lack of coordinated and integrated care opportunities are not overcome using telehealth alone. The need for adjunct services to support telehealth, to connect to local community services and to expand the range of services that can be provided [16] means that a virtual care approach is essential.

A simple example involves the process of getting a prescription. A patient may experience the convenience of a telehealth visit but then has to visit the clinic to collect a prescription [17], thereby undoing any benefits gained in reducing travel time and its associated cost. COVID-19 has accelerated the uptake of electronic prescribing in Australia, however the technological challenges inherent in updating and integrating disparate clinical desktop, mobile and pharmacy software may see national-wide implementation delayed. Another example is the use of sensors to monitor vital signs, especially in the management of chronic disease. The continuous data collected does not flow directly to the doctor [18] due to the lack of platforms that enable end-to-end integration. Care fragmentation is quite common when patients receive care from multiple health service providers; what is needed is innovations in electronic medical records and clinical systems that are interoperable enabling seamless data sharing between multiple service providers. One of the main improvements needed is a model of care delivery and clinical systems that integrates consumer technology (e.g. wearable, smartphones, mobile apps, sensors) with electronic medical records that captures remote monitoring data and patient vital signs to give a holistic picture of a patient's health. Improvements in remote monitoring and collection of vital signs outside of clinic visits are critical in the management of mental health and chronic disease, especially in the older population. Some of the main barriers to the uptake of telehealth reported by older participants (mean age $=68$ ) are technical problems, believing that telehealth is not necessary and preference for in-person care [9]. These barriers can be addressed by enabling on-time technical support [9] and delivering educational initiatives to reduce anxiety around technology use. In addition, we have seen anecdotal reports on the older adults willingly embracing technology during the pandemic motivated by the desire to stay connected with family and friends. 


\section{Beyond telehealth: opportunities to address recurring healthcare pain points}

Australian healthcare faces many recurring challenges in delivering care (Figure 1). These challenges fall in to two broad categories: those related to the context of care delivery (A-C) and those related to the logistics associated with the delivery of care (E$\mathrm{G).}$

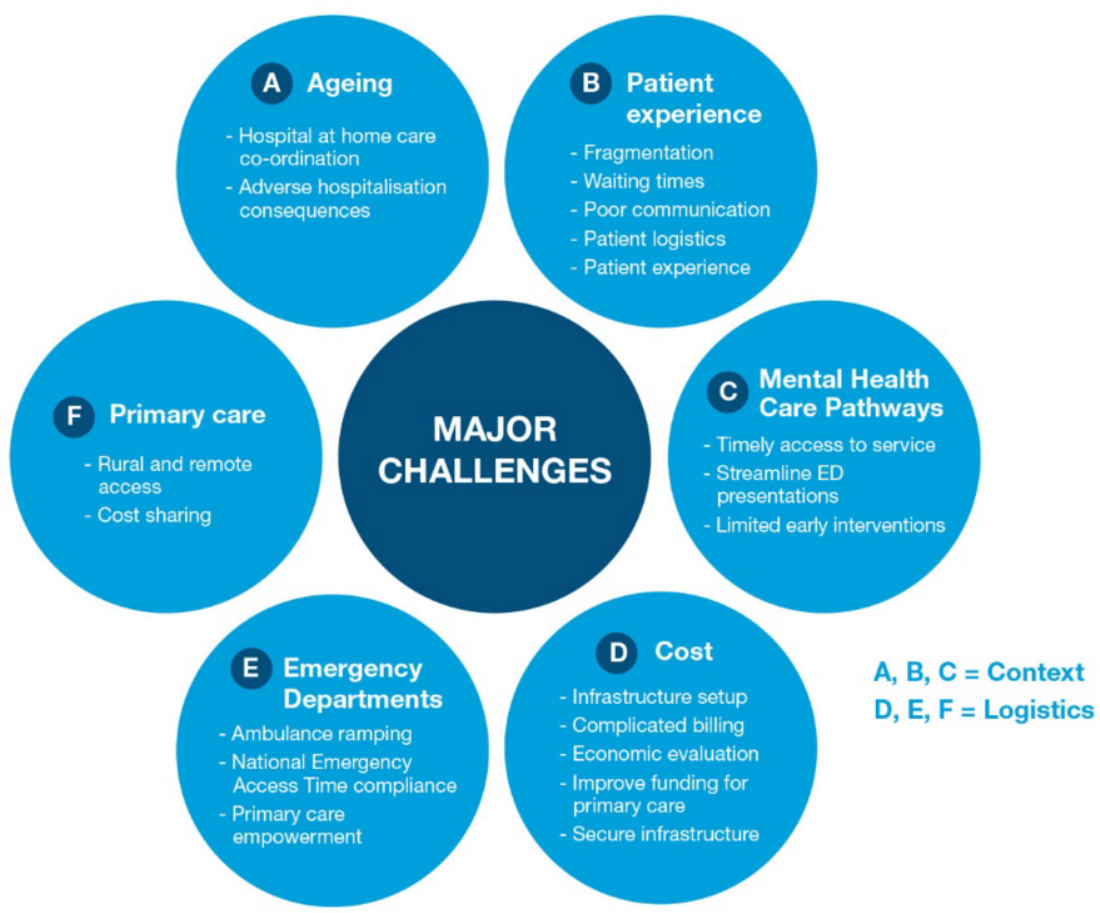

Figure 1. The recurring challenges in healthcare delivery in Australia [19].

There is an ongoing need to improve poor health outcomes in rural and remote communities in Australia through improved access to health services. The time is ripe to build upon the significant achievements in relation to innovative models of Primary Health Care since the first National Rural Health Strategy [14]. Often these isolated rural and remote communities are too small to support traditional models of health delivery locally, so residents must access care from larger urban centres. Unfortunately, access to health services provided in larger centres remains a problem for many residents of isolated settlements [14]. Essentially what is needed is a change at policy level to enable the adoption of patient-centered primary and community care [20] and a review of primary care cost model.

Telehealth services in rural Australia has been supported by the Medicare Benefits Scheme (MBS) for psychiatry since 2003, for specialist services since 2011, and for rural GP services since 2019. In response to the COVID-19 pandemic, the Australian Government extended the funding for telehealth services to consultations between patients and their general practitioners, mental health providers and medical specialists, 
and to midwives. It is now considering how telehealth can be used by any healthcare provider (including allied health) in the future. The opportunity to investigate how virtual care could impact costs of the healthcare system in Australia is a vital piece in improving patient outcomes whatever the drivers, be they patient demand, pandemics, or embracing new care pathways enabled by technology. Given the complex nature of the Australian healthcare funding model, cooperatively operated by all levels of Australian government - federal, state and territory, and local. Added to this, maintaining world-class, safe and affordable healthcare for all Australians, means we need to look for new and alternative, data and technology supported, ways to shift (rather than transform) healthcare in Australia.

The rise of COVID-19 has not diminished the normal demand for the health system to manage chronic diseases and emergency situations. There needs to be a continued focus to keep people with chronic illness and comorbidities healthy and out of hospital. Coupled with the need for social distancing, public and private funding models need to expand to fund doctors, nurses and allied health professionals to deliver care, where clinically appropriate, virtually. These measures support people who require medical care, who may have high levels of anxiety due to the crisis, to seek treatment and care as well as help to protect transmission of the disease through the healthcare workforce [21]. Delivering care virtually by keeping clinically appropriate patients out of acute care settings will prevent negative patient outcomes associated with ambulance ramping and prolonged waiting times to receive treatment at emergency departments.

There are numerous opportunities to improve health care service provision for older patients via virtual care. Remote care and monitoring for older patients will prevent unnecessary hospitalization and reduce the occurrence of delirium which commonly sets in hospital environment, adverse events from medication errors, falls associated with confusion and polypharmacy. One of the biggest advances comes from real-time monitoring as seen in the reductions of severe hypoglycemia and improved quality of life [22].

There are several pain points for mental health patients presenting at emergency departments, such as prolonged waiting times due to mental health bed shortage, increased hospital length of stay, which is disproportionate to their lower acuity level, increased use of restrictive intervention and more than half of the presentations are repeat presentations [23]. The pandemic has seen a surge in telehealth mental health service delivery which has dramatically reduced the strain on the healthcare system. Although adverse events such as suicide has not increased in Australia as a result of the pandemic, the real effect of the pandemic is yet to be seen in years to come, and requires long term preparedness by going beyond video and tele consult. Opportunities in virtual care will arise from technological innovation in integration of consumer wearable, remote monitoring, and early intervention alert system for crisis management.

\section{Move to patient centered care}

The benefits to patients and supporting patient-centered care, particularly for routine treatment of chronic conditions is clear $[15,18]$. This is supported by the increasing set of digital services and health monitoring that can be at home [24, 25]. This leads to healthcare delivered more conveniently for the patient, often eliminating travel time and costs and minimizing waiting times. Also, of considerable benefit to the patient, is the ability to include multiple healthcare providers, allied health providers and family and 
carers in one consultation. This encapsulates a more personalized and proactive approach to care for the patient, thus potentially resulting improved experience and outcomes.

From connecting by video to virtual reality, augmented reality and extended reality for education and simulation, to therapeutics within the operating room are all examples of innovation in virtual care which are centered around providing better care outcomes for the patients. For those of us fortunate enough to be living within first-world healthcare systems, brick and mortar hospitals will become more decentralized over the next five years. It is already happening in the UAE and with Kaiser Permanente. Healthcare provider in these examples already have many of their patients using wearables, transmitting data to providers via the cloud and patient data can be accessed from anywhere on demand.

\section{Potential solution}

To achieve a new and effective virtual care environment demands innovative and comprehensive solutions to integrate and blend technologies to improve the experience. As demonstrated in Figure 2, these solutions need to be scalable, interoperable, and use secure infrastructure. More than this, they need to incorporate how patients and providers can use these solutions, integrate into clinical and care processes, as well as make effective use of existing technology. What is needed is a longer-term perspective to improve clinical care models, improve the patient experience, reduce costs and improve patient safety. The use of augmented reality and virtual reality in consultations could provide a much richer experience.

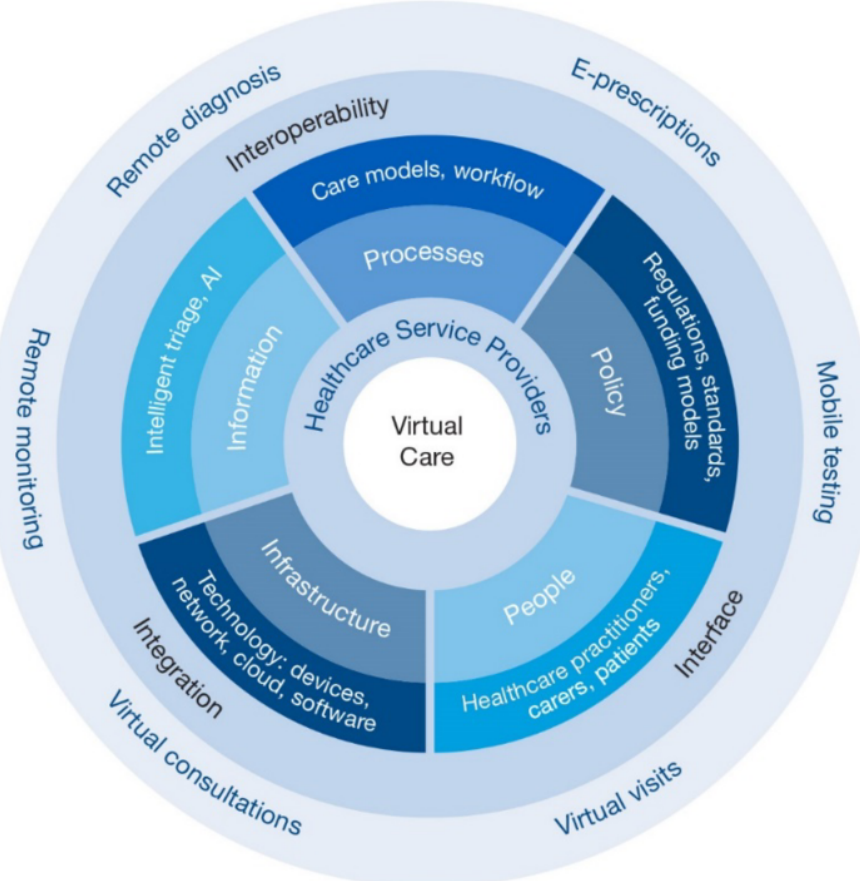

Figure 2. The construction of virtual care and its components [19] 
Solutions need the combination and integration of technology, care models, workflow, workforce skills, funding models, policy, standards and guidance. Since 2009 over 80 commercial telemedicine companies have entered the market [26], with the majority catering for low-acuity issues. However, many of these solutions do not use primary care physicians so there is disconnect between commercial solutions and usual pathways of care. Whilst the use of telehealth services was projected to increase in other countries, in Australia prior to COVID-19 less than 0.1\% of Medicare Benefits Scheme (MBS) consultations were via teleconsultation. Any potential solution will need to see a shift in funding models and virtual care healthcare service incentivized. The solutions must incorporate safety and quality, standards of care, patient engagement, continuity of care, privacy and security.

\section{Conclusion}

Virtual care should be considered as a supplement to face-to-face and telehealth services. It will provide additional choice for patients about when, where and how they seek healthcare that suits their needs. We need to move from the theoretical construction of what virtual care can provide to the reality of creating a healthcare system that implements and supports virtual care. Currently, there is no framework for how to move forward with virtual care in a comprehensive manner in Australia [20, 27]. Without an inclusive and all-encompassing framework for understanding what virtual care is, the facets it comprises, its challenges and what benefits it can realize, it is not possible to innovate and devise solutions that meet the changing and competing demands in Australian healthcare including measures to address digital divide which must be addressed as part of regional economic and social development strategy [28]. This becomes a powerful driver for investigating the strategic, tactical and operational necessities to further patient-centred and integrated care which will reduce financial burden for our healthcare system by tackling common pain-points such as minimizing length of stay and last-minute cancellation [29]. Despite the barriers, the drivers for exploring solutions using virtual care outweigh the perceived difficulties. Virtual care is not anticipated to replace traditional models of care, but rather to enhance them and there is a need to substantially integrate virtual care into routine care practices in Australia.

\section{Acknowledgement}

"This research and Virtual Care White Paper was funded by Cisco Systems Australia Pty Ltd through Cisco's Country Digital Acceleration program."

\section{References}

[1] Schwamm LH, Erskine A, Licurse A, A digital embrace to blunt the curve of COVID19 pandemic. NPJ Digit Med, 2020. 3: p. 64.

[2] Australian Government. The Department of Health - Telehealth. 2020.

[3] ISO, ISO/TR 14639-1 Health Informatics - Capacity-based eHealth architecture roadmap. 2012.

[4] ISO, ISO/TR 16056, 16058 Health Informatics - Interoperability of telehealth systems and networks, Interoperability of tele-learning systems. 2004. 
[5] Van Velthoven MH, Cordon C. Sustainable Adoption of Digital Health Innovations: Perspectives From a Stakeholder Workshop. Journal of medical Internet research, 2019 21(3): p. e11922-e11922.

[6] Jamieson T, et al. Virtual Care: A Framework for a Patient-Centric System, Women's College Hospital. 2015. 21.

[7] Shaw J, et al. Virtual care policy recommendations for patient-centred primary care: findings of a consensus policy dialogue using a nominal group technique. J Telemed Telecare, 2018. 24(9): p. 608615 .

[8] Fronczek AE. Nursing Theory in Virtual Care. Nursing science quarterly, 2019. 32(1): p. 35-38.

[9] Gorst SL, et al. Home telehealth uptake and continued use among heart failure and chronic obstructive pulmonary disease patients: A systematic review. Ann Behav Med, 2014. 48.

[10] Finkelstein SM, Speedie SM, Potthoff S. Home telehealth improves clinical outcomes at lower cost for home healthcare. Telemed J E Health, 2006. 12(2): p. 128-36.

[11] Rosenfeld S. Patients, Clinicians Satisfied with Telehealth for Follow-Up Care. Inside Digital Health 2019; Available from: https://www.idigitalhealth.com/news/patients-clinicians-satisfied-withtelehealth-for-followup-care?rel=0.

[12] Chandrashekar P, Jain SH. Eliminating Barriers to Virtual Care: Implementing Portable Medical Licensure. American Journal of Managed Care, 2020. 26(1): p. 20-22.

[13] Carey TA, et al. What is the overall impact or effectiveness of visiting primary health care services in rural and remote communities in high-income countries? A systematic review. BMC Health Serv. Res., 2018. 18(1).

[14] Kuipers W, et al. Primary health care delivery models in rural and remote Australia - a systematic review. 2010.

[15] Frakt AB, Chernew ME. The Importance of Relative Prices in Health Care Spending. JAMA, 2018. 319(5): p. 441-442.

[16] Bywood PT, Katterl R, Lunnay BK. Strategies to reduce barriers and inequities in access to health care services for rural/remote areas. 2016: Primary Health Care Research and Information Service.

[17] MacKlenzie M, Smith A, Nespolon H. Are we ready for the telehealth revolution?, in Life Matters, ABC Radio. 2020

[18] Oran DP, Topol EJ, The rise of the virtualist. The Lancet, 2019. 394(10192).

[19] Williams PAH, Perimal-Lewis L, Mudd G, Gunasekara G. Reimagining a better healthcare system through virtual care (2020) 36. $\quad$ Retrieved from https:/www.cisco.com/c/dam/en_us/solutions/industries/resources/healthcare/reimagining-betterhealthcare-system-through-virtual-care.pdf.

[20] Shaw J, et al. Virtual care policy recommendations for patient-centred primary care: findings of a consensus policy dialogue using a nominal group technique. Journal of telemedicine and telecare, 2018. 24(9): p. 608-615.

[21] Angus D, Connolly M, Salita M. The shift to virtual care in response to COVID-19. PWC Australia 2020; Available from: https://www.pwc.com.au/important-problems/coronavirus-covid-19/shiftvirtual-care-response.html

[22] Polonsky WH, Peters AL, Hessler D. The Impact of Real-Time Continuous Glucose Monitoring in Patients 65 Years and Older. Journal of diabetes science and technology, 2016. 10(4): p. 892-897.

[23] Daniel C, et al. Characteristics and clinical outcomes for mental health patients admitted to a behavioural assessment unit: Implications for model of care and practice. International Journal of Mental Health Nursing (2020)

[24] Abani P. Medical Grade EKG in Your Pocket. 2012-2020 [cited 2020; Available from: https://www.alivecor.com/press.

[25] Sullivan M. Telehealth's future is bright. Here's what it'll look like in 2025. (COVID-19 could prove to be telemediciine's watershed moment. Fast Company Magazine, Startup Report 2020 [cited 2020 May 17]; Available from: https://www.fastcompany.com/90505924/telehealths-future-is-bright-heres-whatitll-look-like-in-2025.

[26] Anderson J, Ganguli I. Unpacking the Potential for Virtual Care. J Gen Intern Med, 2019. 34(12): p. 2906-2907.

[27] Waller M, Stotler C. Telemedicine: a Primer. Curr Allergy Asthma Rep, 2018. 18(10): p. 54.

[28] Afshar Ali M, Alam K, Taylor B. Do social exclusion and remoteness explain the digital divide in Australia? Evidence from a panel data estimation approach. Economics of Innovation and New Technology. 2020;29(6):643-59.

[29] Tam A, Leung A, O'Callaghan C, Fagermo N. Role of Telehealth in Perioperative Medicine for Regional and Rural Patients in Queensland. Internal Medicine Journal. 47.8 (2017): 933-37. 